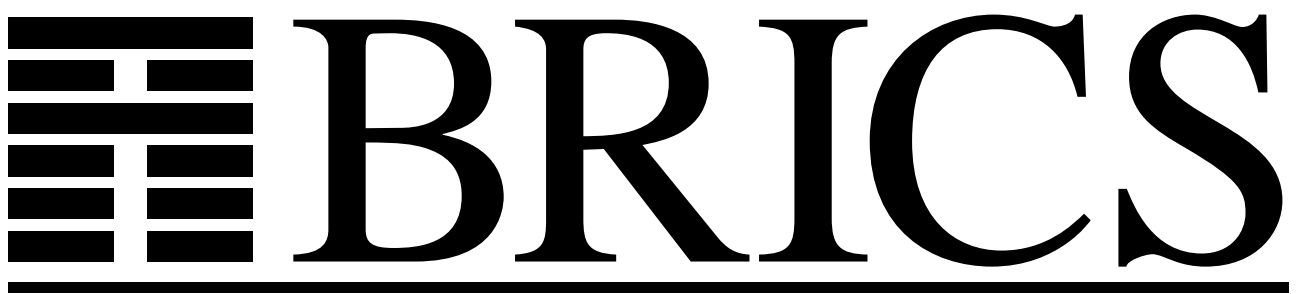

Basic Research in Computer Science

\title{
A Rational Deconstruction of Landin's J Operator
}

Olivier Danvy

Kevin Millikin 
Copyright (c) 2006, Olivier Danvy \& Kevin Millikin.

BRICS, Department of Computer Science

University of Aarhus. All rights reserved.

Reproduction of all or part of this work is permitted for educational or research use on condition that this copyright notice is included in any copy.

See back inner page for a list of recent BRICS Report Series publications. Copies may be obtained by contacting:

\author{
BRICS \\ Department of Computer Science \\ University of Aarhus \\ IT-parken, Aabogade 34 \\ DK-8200 Aarhus N \\ Denmark \\ Telephone: +4589429300 \\ Telefax: $\quad+4589425601$ \\ Internet: BRICS@brics.dk
}

BRICS publications are in general accessible through the World Wide Web and anonymous FTP through these URLs:

http://www.brics.dk

ftp: //ftp.brics.dk

This document in subdirectory RS / $06 / 4$ / 


\title{
A Rational Deconstruction of Landin's J Operator*
}

\author{
Olivier Danvy and Kevin Millikin \\ BRICS $^{\dagger}$ \\ Department of Computer Science \\ University of Aarhus ${ }^{\ddagger}$
}

February 28, 2006

\begin{abstract}
Landin's J operator was the first control operator for functional languages, and was specified with an extension of the SECD machine. Through a series of meaning-preserving transformations (transformation into continuation-passing style (CPS) and defunctionalization) and their left inverses (transformation into direct style and refunctionalization), we present a compositional evaluation function corresponding to this extension of the SECD machine. We then characterize the J operator in terms of CPS and in terms of delimited-control operators in the CPS hierarchy. Finally, we present a motivated wish to see Landin's name added to the list of co-discoverers of continuations.
\end{abstract}

\footnotetext{
${ }^{*}$ Extended version of an article to appear in the proceedings of the 17th International Workshop on the Implementation and Application of Functional Languages (IFL'05), Dublin, Ireland, September 2005.

${ }^{\dagger}$ Basic Research in Computer Science (www.brics.dk),

funded by the Danish National Research Foundation.

${ }^{\ddagger}$ IT-parken, Aabogade 34, DK-8200 Aarhus N, Denmark.

Email: <danvy@brics.dk>, <kmillikin@brics.dk>
} 


\section{Contents}

1 Introduction $\quad 1$

1.1 Deconstruction of the SECD machine with the J operator . . . . 1

1.2 Prerequisites and domain of discourse . . . . . . . . . . . 2

1.3 Overview ........................ 4

2 Deconstruction of the SECD machine with the J operator 4

2.1 The starting specification . . . . . . . . . . . . . 4

2.2 A disentangled specification . . . . . . . . . . . . . . 5

2.3 A higher-order counterpart . . . . . . . . . . . . . 6

2.4 A stack-less, caller-save counterpart . . . . . . . . . . . . 7

2.5 A dump-less direct-style counterpart . . . . . . . . . . . . . . 8

2.6 A control-less direct-style counterpart . . . . . . . . . . . . . . . 9

2.7 A compositional counterpart . . . . . . . . . . . . . . . 10

2.8 Summary ........................... 11

3 Three simulations of the J operator $\quad 11$

4 Four more simulations of the J operator $\quad 12$

4.1 The $\mathcal{C}$ operator and the CPS hierarchy . . . . . . . . . . . . . . 12

4.2 The call/cc operator and the CPS hierarchy . . . . . . . . . 13

5 On the design of control operators $\quad 13$

6 Related work $\quad 14$

6.1 Landin and Burge . . . . . . . . . . . . . . . . . . . . . 14

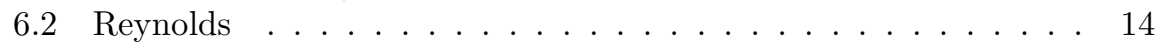

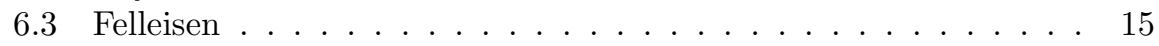

6.4 Felleisen and Burge . . . . . . . . . . . . . . 16

7 An alternative deconstruction $\quad \mathbf{1 6}$

7.1 Our starting point: Burge's specification . . . . . . . . . . . . 16

7.2 Burge's specification in defunctionalized form . . . . . . . . . . . 17

7.3 A higher-order counterpart . . . . . . . . . . . . . . 18

7.4 The rest of the rational deconstruction . . . . . . . . . . . 19

7.5 Three alternative simulations of the J operator . . . . . . . . . . 20

7.6 Related work . . . . . . . . . . . . . . 21

8 Summary and conclusion $\quad 21$

9 On the origin of first-class continuations $\quad 22$ 


\section{Introduction}

Forty years ago, Peter Landin unveiled the first control operator, J, to a heretofore unsuspecting world [26-28]. He did so to generalize the notion of jumps and labels and showed that the resulting notion of 'program closure' makes sense not just in an imperative setting, but also in a functional one. He specified the $\mathrm{J}$ operator by extending the SECD machine [25].

At IFL'04, Danvy presented a 'rational deconstruction' of Landin's SECD machine into a compositional evaluation function [9]. The goal of this work is to extend this rational deconstruction to the $\mathrm{J}$ operator.

\subsection{Deconstruction of the SECD machine with the J op- erator}

Let us outline our deconstruction of the SECD machine before substantiating it in Section 2. We essentially follow the order of Danvy's deconstruction [9], though with a twist: in the middle of the derivation, we abandon the stack-threading, callee-save features of the SECD machine for the more familiar register-based, caller-save features of traditional definitional interpreters $[19,31$, $34]$.

The SECD machine is defined as the transitive closure of a transition function over a quadruple - a data stack containing intermediate values (of type $S$ ), an environment (of type E), a control stack (of type C), and a dump (of type D):

run : $\mathrm{S} * \mathrm{E} * \mathrm{C} * \mathrm{D} \rightarrow$ value

The definition of this transition function is complicated because it has several induction variables, i.e., it dispatches on several components of the quadruple.

- We disentangle it into four transition functions, each of which has one induction variable, i.e., dispatches on one component of the quadruple:

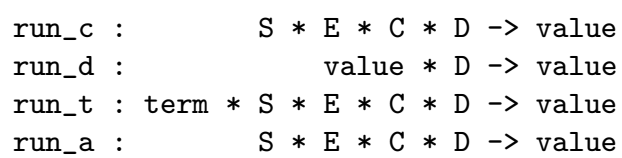

The first function, run_c, dispatches towards run_d if the control stack is empty, run_t if the top of the control stack contains a term, and run_a if the top of the control stack contains an apply directive. This disentangled specification is in defunctionalized form: the control stack and the dump are defunctionalized data types, and run_c and run_d are the corresponding apply functions.

- Refunctionalization eliminates the two apply functions:

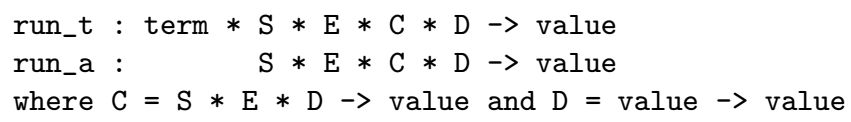


As identified in the first rational deconstruction [9], the resulting program is a stack-threading, callee-save interpreter in continuation-passing style (CPS).

- In order to focus on the nature of the $\mathrm{J}$ operator, we eliminate the data stack and adopt the more familiar caller-save evaluation strategy:

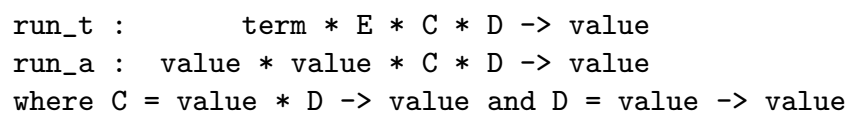

The interpreter is still in CPS.

- The direct-style transformation eliminates the dump continuation:

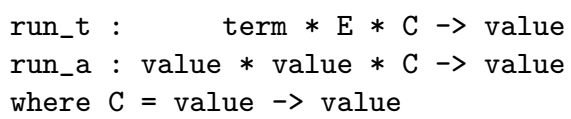

The clause for the $\mathrm{J}$ operator and the main evaluation function are expressed using the delimited-control operators shift and reset [10]. The resulting evaluator still threads an explicit continuation, even though it is not tail-recursive.

- The direct-style transformation eliminates the control continuation:

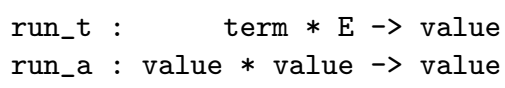

The clauses catering for the non-tail-recursive uses of the control continuation are expressed using the delimited-control operators shift 1 , reset $_{1}$, shift $_{2}$, and reset $_{2}[4,10,14,23,33]$. The resulting evaluator is in direct style. It is also in closure-converted form: the applicable values are a defunctionalized data type and run_a is the corresponding apply function.

- Refunctionalization eliminates the apply function:

run_t : term $* E \rightarrow$ value

The resulting evaluator is compositional.

There is plenty of room for variation in the present reconstruction. The path we are taking seems reasonably pedagogical - in particular, the departure from threading a data stack and managing the environment in a callee-save fashion. Each of the steps is reversible: one can CPS-transform and defunctionalize an evaluator into an abstract machine $[1-4,9]$.

\subsection{Prerequisites and domain of discourse}

Up to Section 2.4, we use pure ML as a meta-language. We assume a basic familiarity with Standard ML and with reasoning about ML programs as well as an elementary understanding of defunctionalization $[13,34]$, the CPS transformation [10,11, 19,31,34,37], and delimited continuations [4, 10, 14, 17, 23]. From Section 2.5, we use pure ML with delimited-control operators. 


\subsection{Overview}

We first detail the deconstruction of the SECD machine into a compositional evaluator in direct style (Section 2). We then analyze the J operator (Sections 3 and 4), review related work (Sections 6 and 5), and conclude (Sections 8 and 9).

\section{Deconstruction of the SECD machine with the $\mathbf{J}$ operator}

\subsection{The starting specification}

Several formulations of the SECD machine with the J operator have been published $[6,16,27]$. We take the most recent one, i.e., Felleisen's [16], as our starting point, and we consider the others in Section 6 .

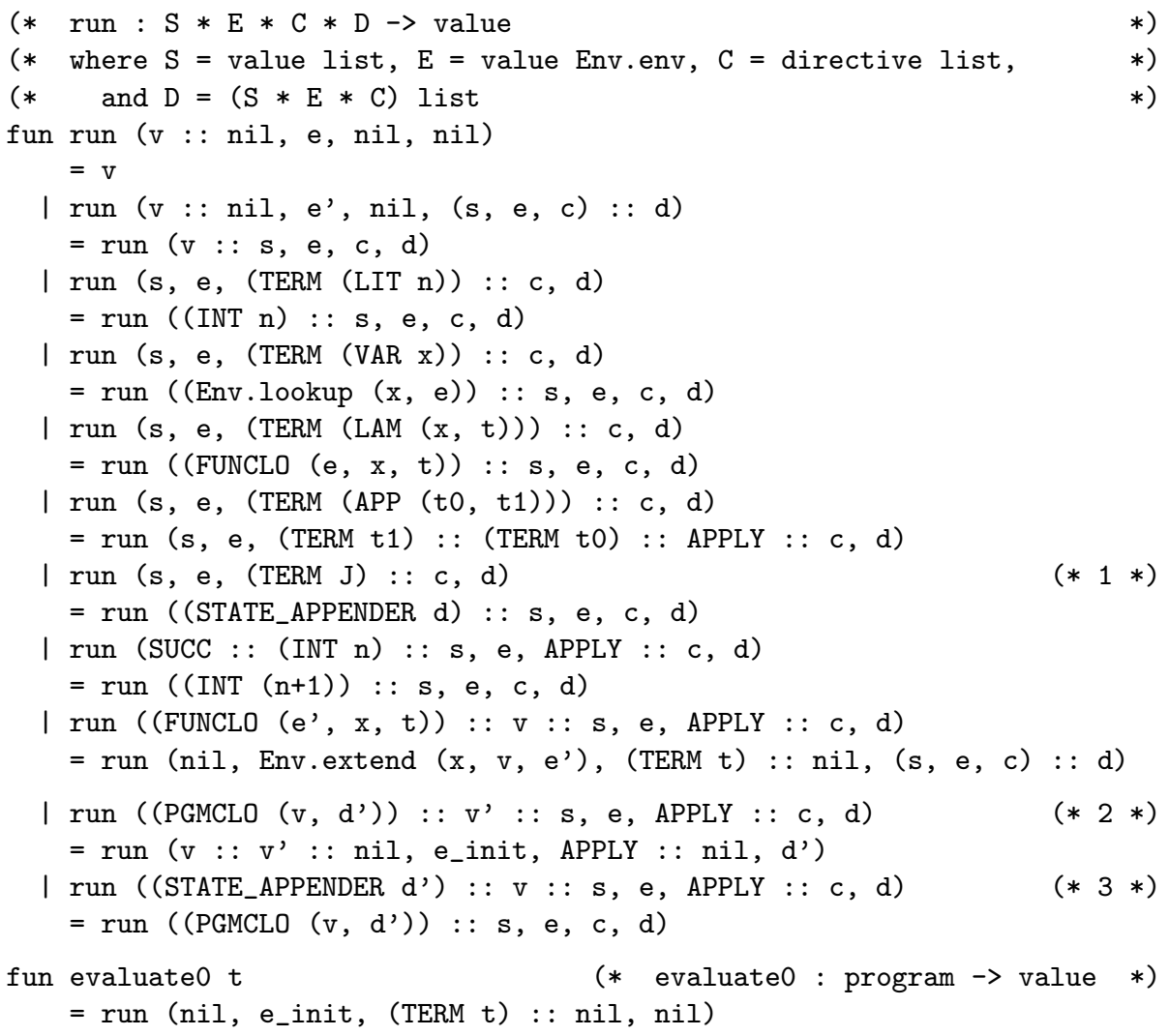

The SECD machine does not terminate for divergent source terms. If it becomes stuck, an ML pattern-matching error is raised (alternatively, the codomain of run could be made value option and a fallthrough else clause could be added). Otherwise, the result of the evaluation is $\mathrm{v}$ for some ML value $\mathrm{v}$ : value. The clause marked "1" specifies that the J operator, at any point, denotes the current dump; evaluating it captures this dump and yields a state appender that, when applied (in the clause marked " 3 "), yields a program closure. Applying a program closure (in the clause marked "2") restores the captured dump. 


\subsection{A disentangled specification}

In the definition of Section 2.1, all the possible transitions are meshed together in one recursive function, run. As in the first rational deconstruction [9], we factor run into four mutually recursive functions, each of them with one induction variable. In this disentangled definition,

- run_c interprets the list of control directives, i.e., it specifies which transition to take according to whether the list is empty, starts with a term, or starts with an apply directive. If the list is empty, it calls run_d. If the list starts with a term, it calls run_t, caching the term in an extra component (the first parameter of run_t). If the list starts with an apply directive, it calls run_a.

- run_d interprets the dump, i.e., it specifies which transition to take according to whether the dump is empty or non-empty, given a valid data stack.

- run_t interprets the top term in the list of control directives.

- run_a interprets the top value in the current data stack.

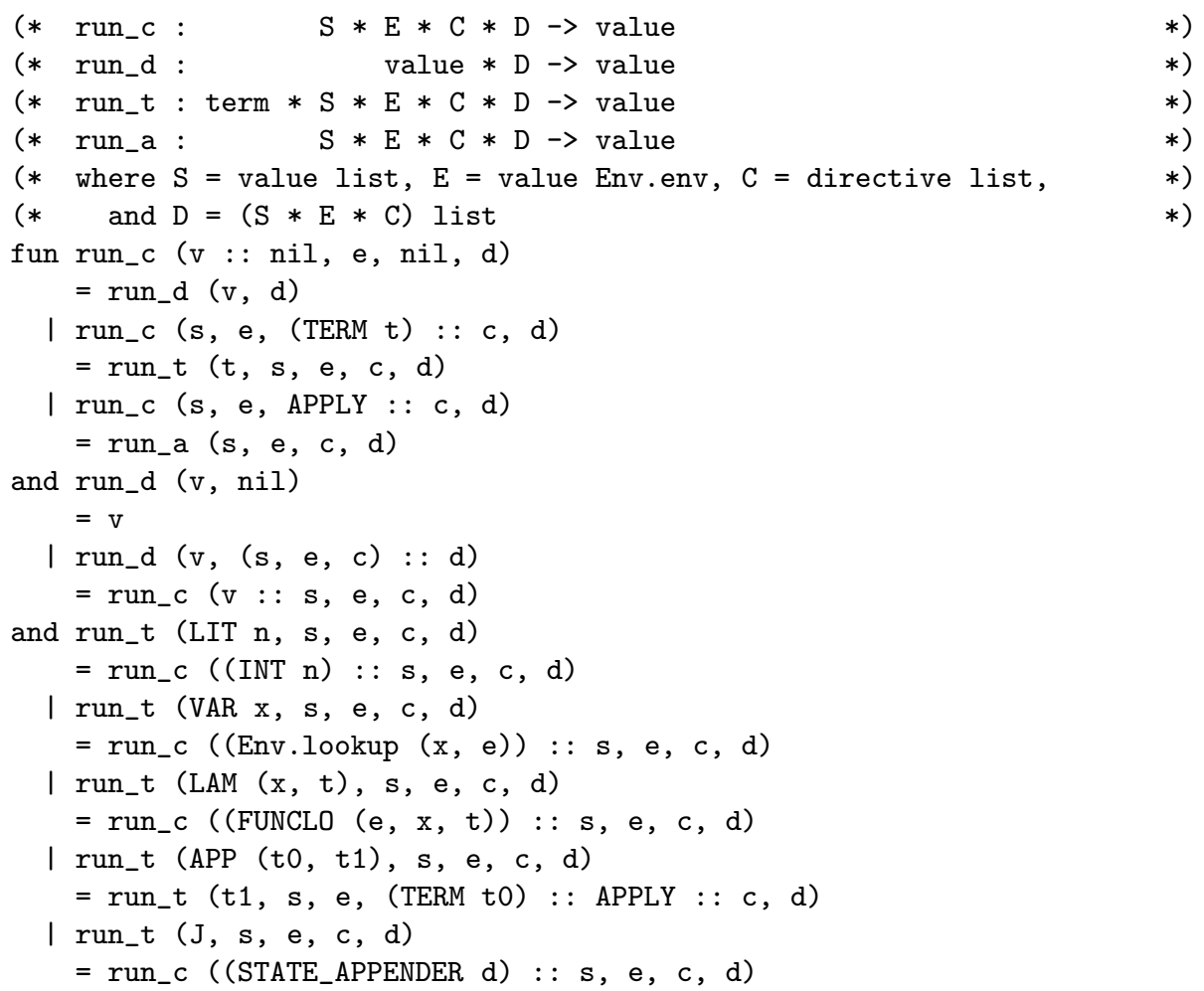




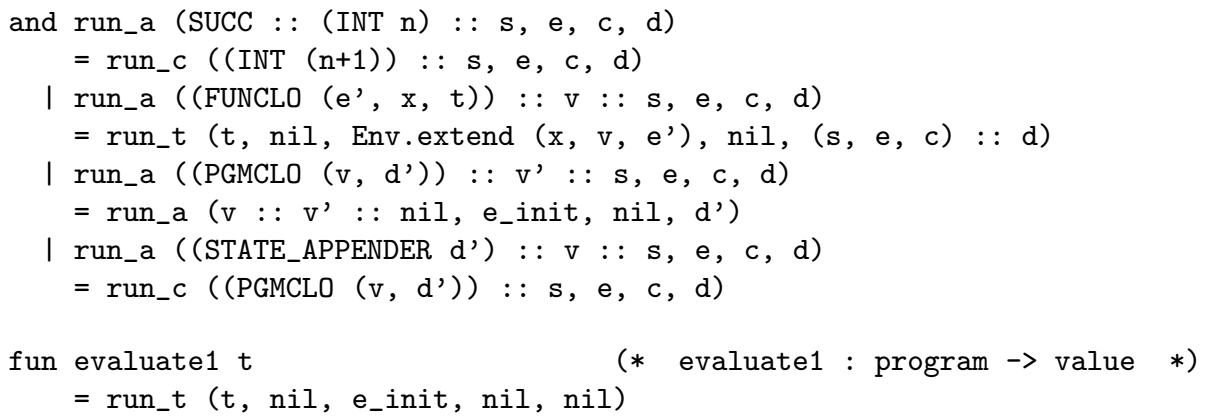

Proposition 1 (full correctness) Given a program, evaluate0 and evaluate1 either both diverge or both yield values that are structurally equal.

\section{$2.3 \quad$ A higher-order counterpart}

In the disentangled definition of Section 2.2, there are two possible ways to construct a dump - nil and consing a triple - and three possible ways to construct a list of control directives - nil, consing a term, and consing an apply directive. (We could phrase these constructions as two data types rather than as two lists.)

These data types, together with run_d and run_c, are in the image of defunctionalization (run_d and run_c are the apply functions of these two data types). The corresponding higher-order evaluator reads as follows; it is higher-order because $\mathrm{c}$ and d now denote functions:

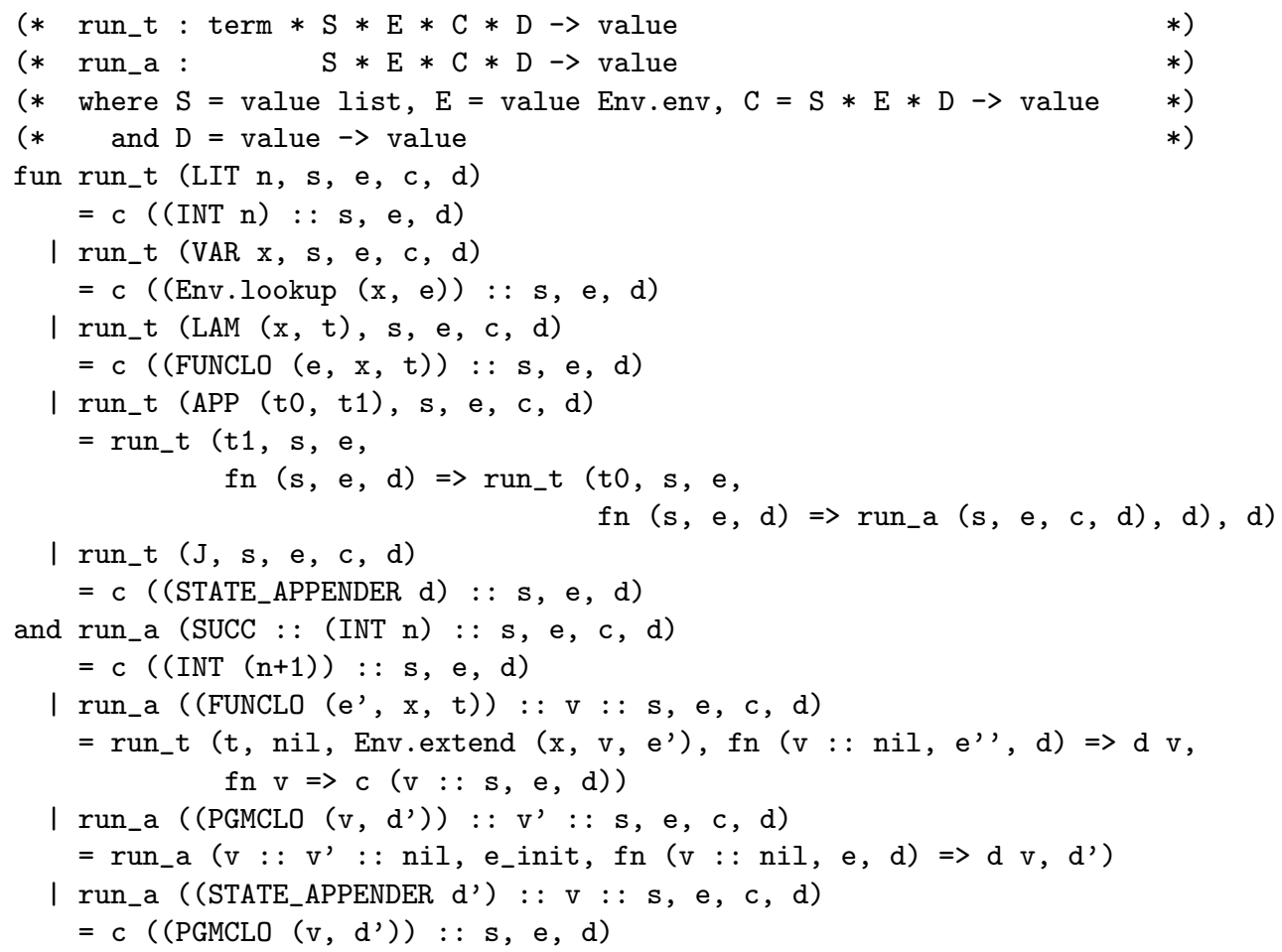


fun evaluate2 $t$

(* evaluate2 : program $\rightarrow$ value $*$ )

$=$ run_t (t, nil, e_init, fn $(v:: n i l, e, d) \Rightarrow d v$, fn $v=>v)$

The resulting evaluator is in CPS, with two layered continuations $c$ and $d$. It threads a stack of intermediate results (s), an environment (e), a control continuation (c), and a dump continuation (d). Except for the environment being callee-save, the evaluator follows a traditional eval-apply schema: run_t is eval and run_a is apply. Defunctionalizing it yields the definition of Section 2.2.

Proposition 2 (full correctness) Given a program, evaluate1 and evaluate2 either both diverge or both yield values that are structurally equal.

\subsection{A stack-less, caller-save counterpart}

We want to focus on J, and the non-standard aspects of the evaluator of Section 2.3 (the data stack and the callee-save environment) are a distraction. We therefore transmogrify the evaluator into the more familiar register-based, caller-save form $[19,31,34]$, renaming run_t as eval and run_a as apply. Intermediate values are explicitly passed instead of being stored on the data stack, and environments are no longer passed to apply and to the control continuation:

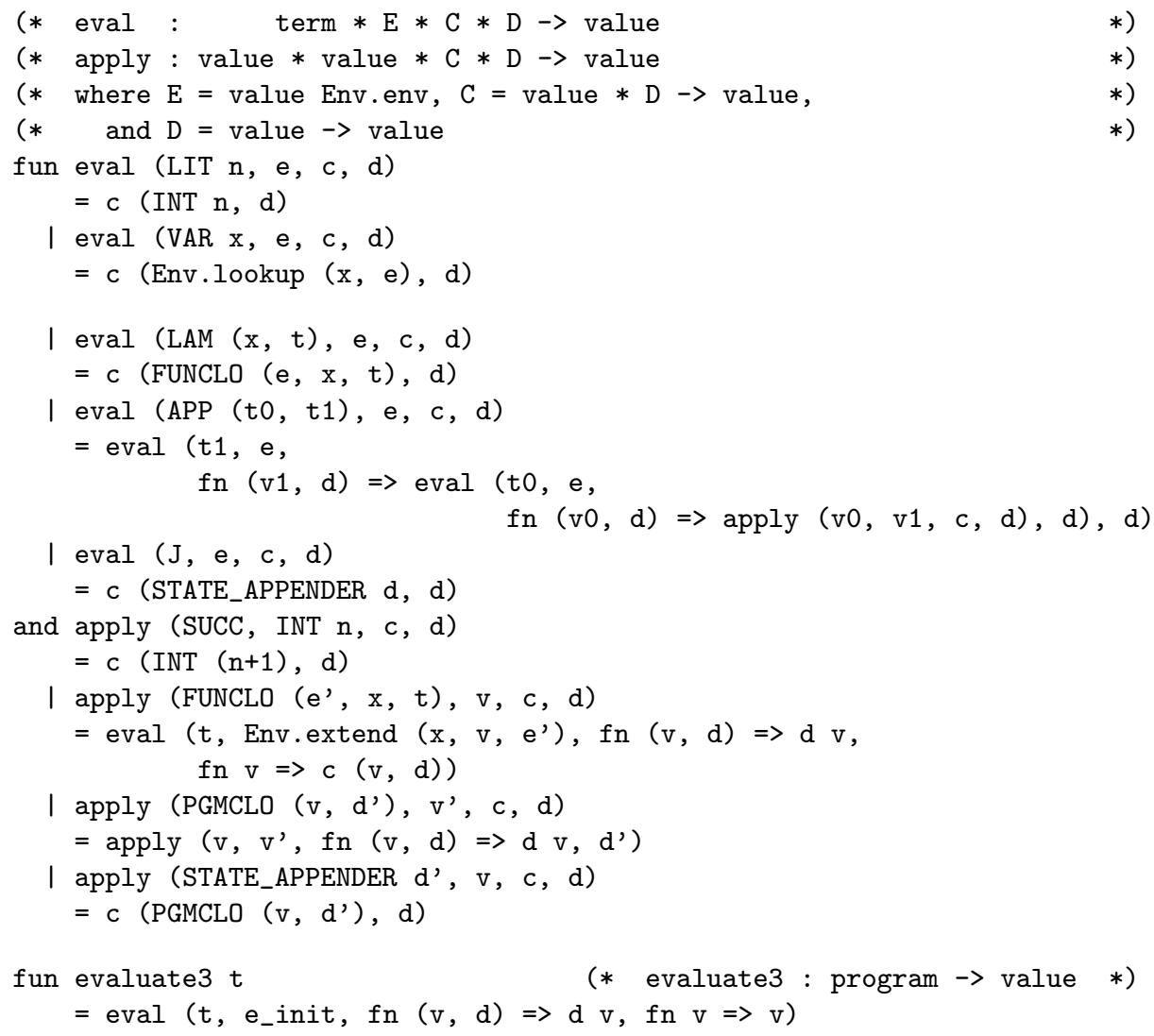

The new evaluator is still in CPS, with two layered continuations. 
Proposition 3 (full correctness) Given a program, evaluate2 and evaluate3 either both diverge or both yield values that are structurally equal.

\subsection{A dump-less direct-style counterpart}

The evaluator of Section 2.4 is in continuation-passing style, and therefore it is in the image of the CPS transformation. The clause for J captures the current continuation (i.e., the dump), and therefore its direct-style counterpart naturally uses call/cc [11]. With an eye on our next step, we do not, however, use call/cc but its cousins shift and reset $[10,14]$ to write the direct-style counterpart.

Concretely, we use an ML functor to obtain an instance of shift and reset with value as the type of intermediate answers [14,17]: reset delimits the (now implicit) dump continuation in evaluate, and corresponds to its initialization with the identity function; and shift captures it in the clauses where $\mathrm{J}$ is evaluated and where a program closure is applied:

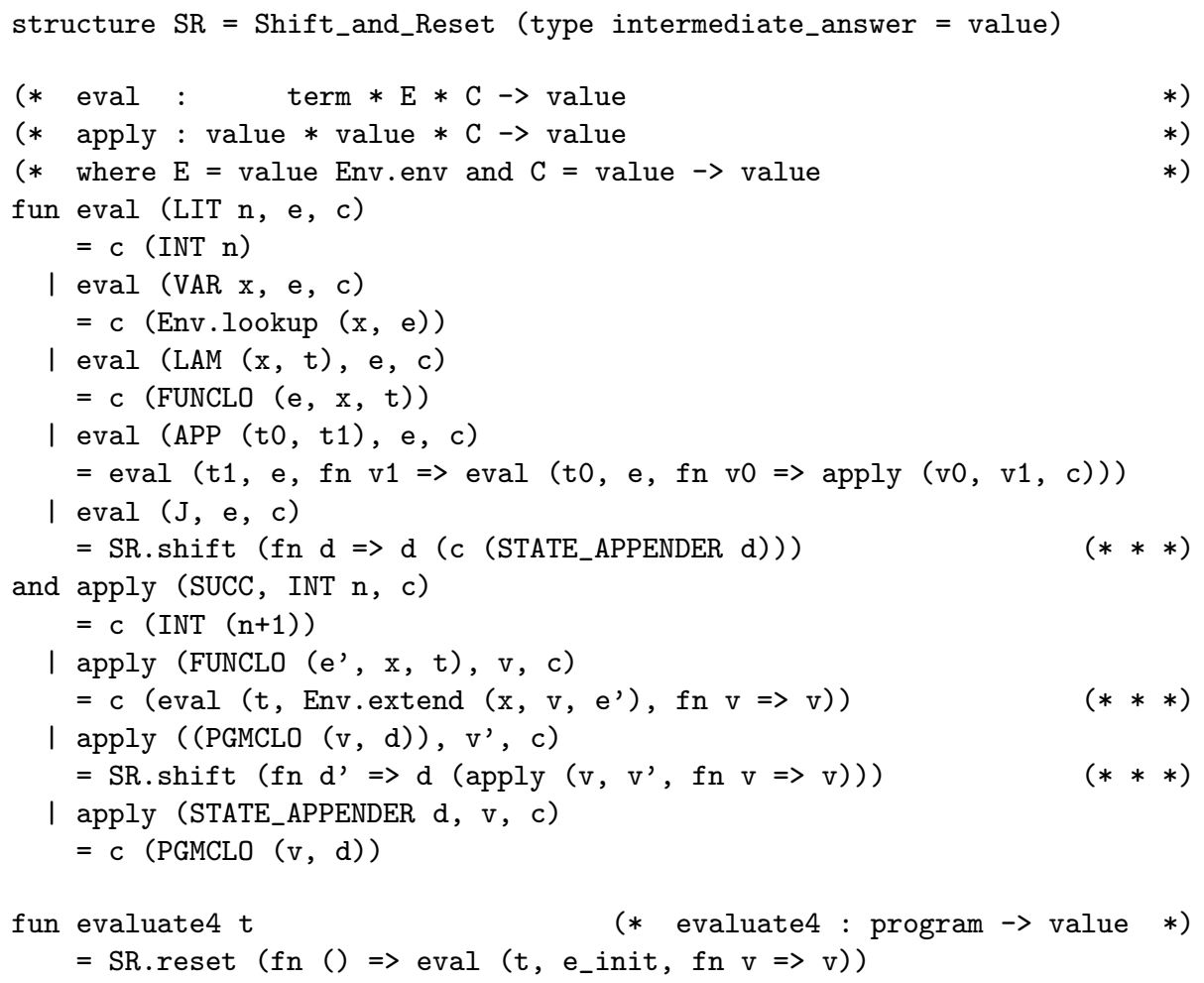

The dump continuation is now implicit and is accessed using shift. CPS-transforming this evaluator yields the evaluator of Section 2.4.

Proposition 4 (full correctness) Given a program, evaluate3 and evaluate4 either both diverge or both yield values that are structurally equal. 


\subsection{A control-less direct-style counterpart}

The evaluator of Section 2.5 still threads an explicit continuation, the control continuation. It however is not in continuation-passing style because of the non-tail calls to c, eval, and apply (in the clauses marked "*") and for the occurrences of shift and reset. This pattern of control is characteristic of the CPS hierarchy $[4,10,14,23]$. We therefore use the delimited-control operators shift $_{1}$, reset $_{1}$, shift 2 , and reset 2 to write the direct-style counterpart of this evaluator ( shift $_{2}$ and reset 2 are the direct-style counterparts of shift 1 and reset $_{1}$, and shift $_{1}$ and reset $_{1}$ are synonyms for shift and reset).

Concretely, we use two ML functors to obtain layered instances of shift and reset with value as the type of intermediate answers $[14,17]$ : $\operatorname{reset}_{2}$ delimits the (now twice implicit) dump continuation in evaluate; shift $_{2}$ captures it in the clauses where $\mathrm{J}$ is evaluated and where a program closure is applied; reset $_{1}$ delimits the (now implicit) control continuation in evaluate and in apply, and corresponds to its initialization with the identity function; and shift $_{1}$ captures it in the clause where $\mathrm{J}$ is evaluated:

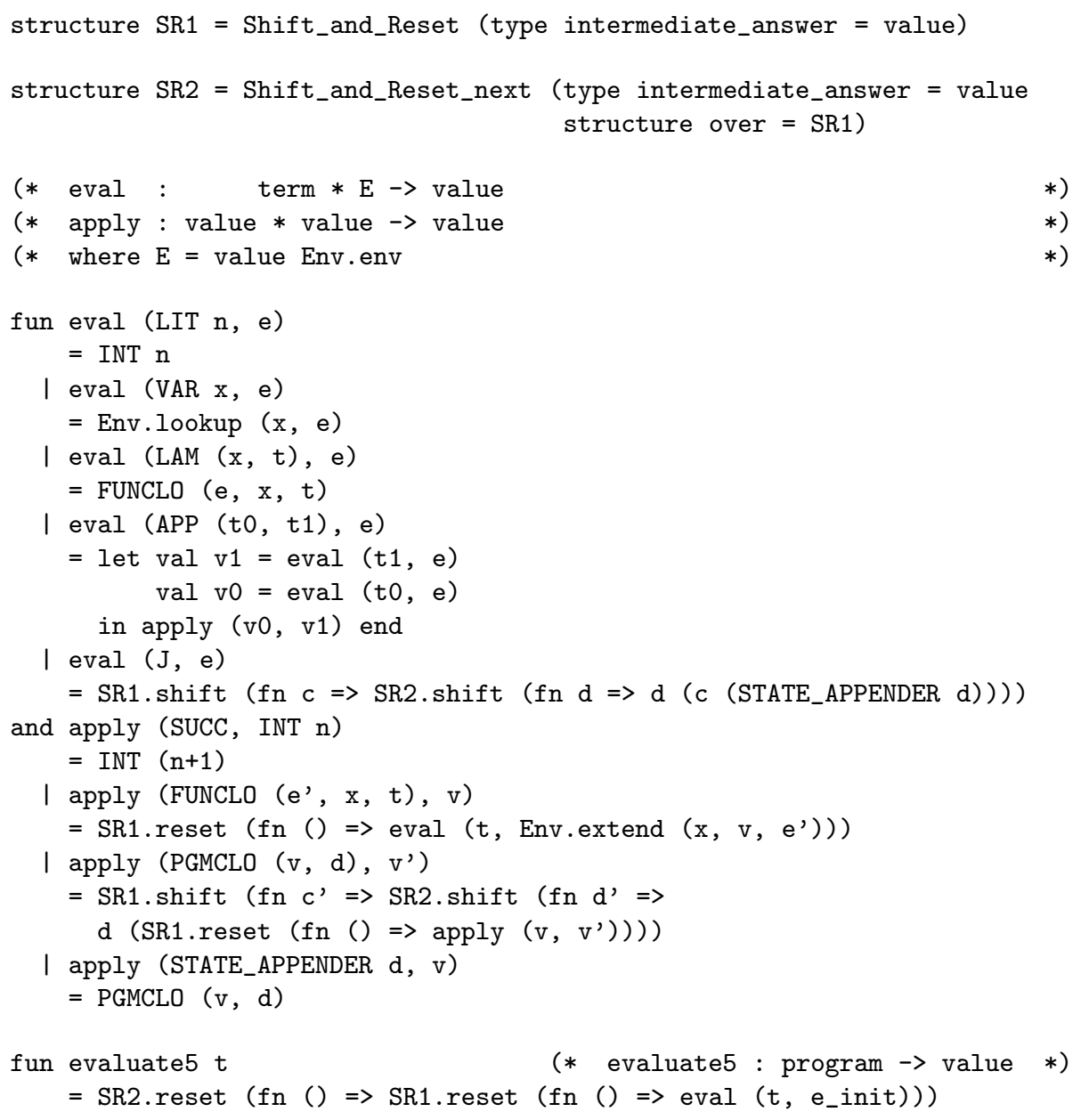


The control continuation is now implicit and is accessed using shift ${ }_{1}$. The dump continuation is still implicit and is accessed using shift ${ }_{2}$. CPS-transforming this evaluator yields the evaluator of Section 2.5.

Proposition 5 (full correctness) Given a program, evaluate4 and evaluate5 either both diverge or both yield values that are structurally equal.

\subsection{A compositional counterpart}

We now turn to the data flow of the evaluator of Section 2.6. As for the SECD machine without $\mathrm{J}$ [9], this evaluator is in defunctionalized form: each of the values constructed with SUCC, FUNCLO, PGMCLO, and STATE_APPENDER are constructed at one place and consumed at another (the apply function). We therefore refunctionalize them into the function space value $\rightarrow$ value:

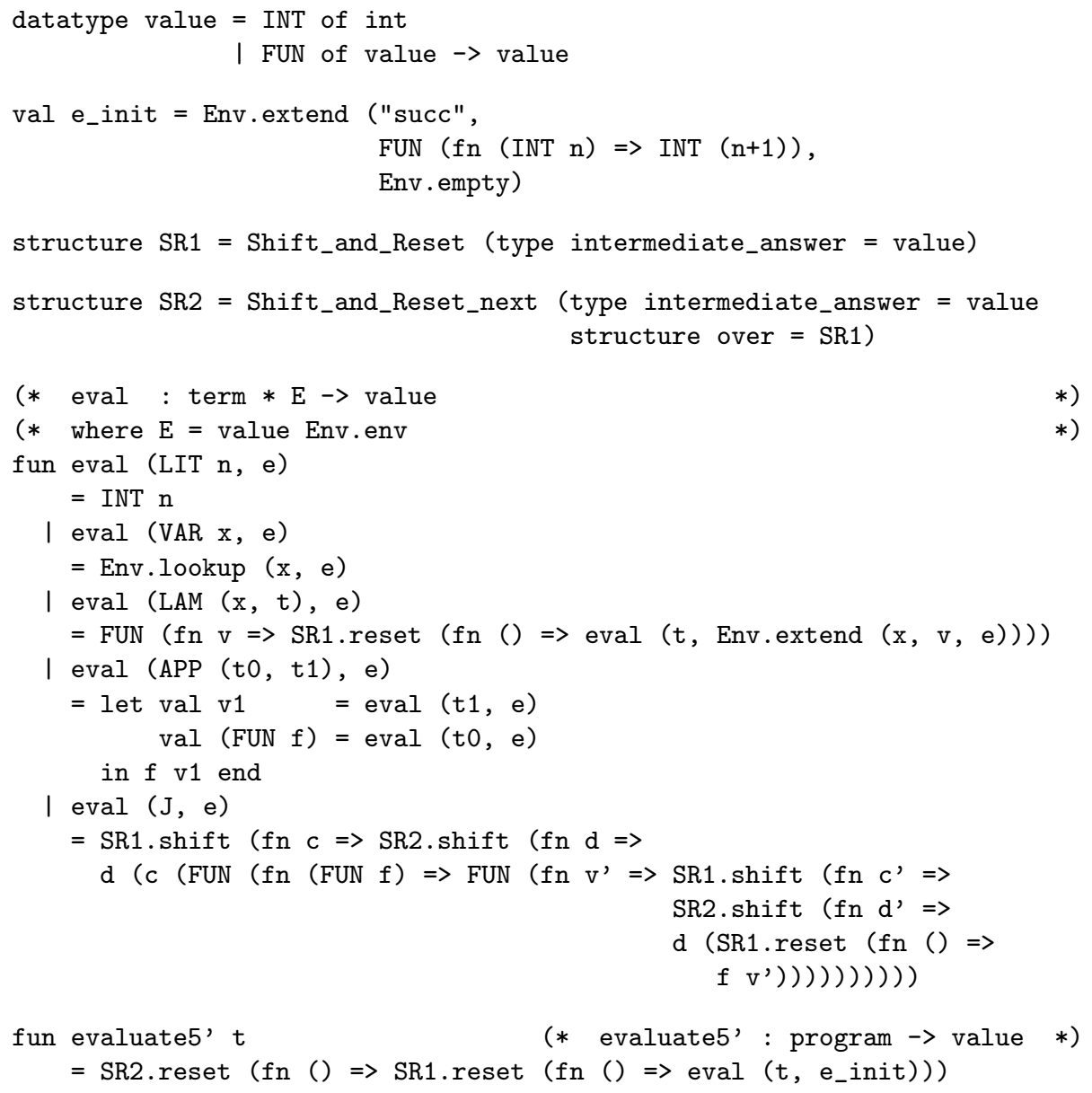

Defunctionalizing this evaluator yields the evaluator of Section 2.6.

Proposition 6 (full correctness) Given a program, evaluate5 and evaluate5' either both diverge or both yield values that are related by defunctionalization. 


\subsection{Summary}

We graphically summarize the derivations as follows. The evaluators in the top row are the defunctionalized counterparts of the evaluators in the bottom row.

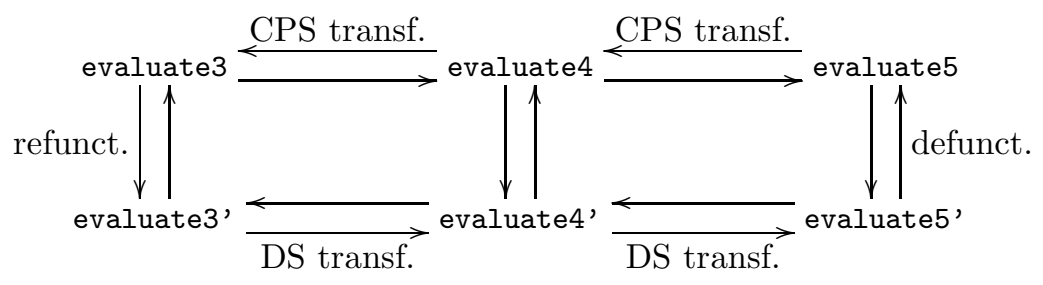

\section{Three simulations of the $\mathbf{J}$ operator}

The evaluator of Section 2.7 and the refunctionalized counterparts of the evaluators of Sections 2.5 and 2.4 are compositional. They can be viewed as syntaxdirected encodings into their meta-language, as embodied in the first Futamura projection [20]. Below, we state these encodings as three simulations of J: one in direct style, one in CPS with one layer of continuations, and one in CPS with two layers of continuations.

We assume a call-by-value meta-language with right-to-left evaluation.

- In direct style, using shift $2\left(\mathcal{S}_{2}\right), \operatorname{reset}_{2}\left(\langle\cdot\rangle_{2}\right), \operatorname{shift}_{1}\left(\mathcal{S}_{1}\right)$, and $\operatorname{reset}_{1}\left(\langle\cdot\rangle_{1}\right)$ :

$$
\begin{aligned}
\llbracket n \rrbracket & =n \\
\llbracket x \rrbracket & =x \\
\llbracket t_{0} t_{1} \rrbracket & =\llbracket t_{0} \rrbracket \llbracket t_{1} \rrbracket \\
\llbracket \lambda x . t \rrbracket & =\lambda x \cdot\langle\llbracket t \rrbracket\rangle_{1} \\
\llbracket \mathbf{J} \rrbracket & =\mathcal{S}_{1} \lambda c . \mathcal{S}_{2} \lambda d . d\left(c \lambda x \cdot \lambda x^{\prime} \cdot \mathcal{S}_{1} \lambda c^{\prime} \cdot \mathcal{S}_{2} \lambda d^{\prime} . d\left\langle x x^{\prime}\right\rangle_{1}\right)
\end{aligned}
$$

A program $p$ is translated as $\left\langle\langle\llbracket p \rrbracket\rangle_{1}\right\rangle_{2}$.

- In CPS with one layer of continuations, using shift $(\mathcal{S})$ and reset $(\langle\cdot\rangle)$ :

$$
\begin{aligned}
\llbracket n \rrbracket^{\prime} & =\lambda c . c n \\
\llbracket x \rrbracket^{\prime} & =\lambda c . c x \\
\llbracket t_{0} t_{1} \rrbracket^{\prime} & =\lambda c . \llbracket t_{1} \rrbracket^{\prime} \lambda x_{1} \cdot \llbracket t_{0} \rrbracket^{\prime} \lambda x_{0} . x_{0} x_{1} c \\
\llbracket \lambda x . t \rrbracket^{\prime} & =\lambda c . c \lambda x . \lambda c . c\left(\llbracket t \rrbracket^{\prime} \lambda x . x\right) \\
\llbracket \mathbf{J} \rrbracket^{\prime} & =\lambda c . \mathcal{S} \lambda d . d\left(c \lambda x . \lambda c . c\left\lfloor\lambda x^{\prime} . \lambda c^{\prime} \cdot \mathcal{S} \lambda d^{\prime} . d\left(x x^{\prime} \lambda x^{\prime \prime} . x^{\prime \prime}\right)\right)\right.
\end{aligned}
$$

A program $p$ is translated as $\left\langle\llbracket p \rrbracket^{\prime} \lambda x . x\right\rangle$.

- In CPS with two layers of continuations (the outer continuation, i.e., the dump continuation, can be $\eta$-reduced in the first three clauses):

$$
\begin{aligned}
& \llbracket n \rrbracket^{\prime \prime}=\lambda c . \lambda d . c n d \\
& \llbracket x \rrbracket^{\prime \prime}=\lambda c . \lambda d . c x d \\
& \llbracket t_{0} t_{1} \rrbracket^{\prime \prime}=\lambda c \cdot \lambda d \cdot \llbracket t_{1} \rrbracket^{\prime \prime}\left(\lambda x_{1} \cdot \lambda d \cdot \llbracket t_{0} \rrbracket^{\prime \prime}\left(\lambda x_{0} \cdot \lambda d \cdot x_{0} x_{1} c d\right) d\right) d \\
& \llbracket \lambda x . t \rrbracket^{\prime \prime}=\lambda c . \lambda d . c\left(\lambda x . \lambda c . \lambda d . \llbracket t \rrbracket^{\prime \prime}(\lambda x . \lambda d . d x) \lambda x . c x d\right) d \\
& \llbracket \mathbf{J} \rrbracket^{\prime \prime}=\lambda c \cdot \lambda d . c\left(\lambda x . \lambda c \cdot \lambda d^{\prime \prime \prime} . c\left(\lambda x^{\prime} \cdot \lambda c^{\prime} \cdot \lambda d^{\prime} \cdot x x^{\prime}\left(\lambda x^{\prime \prime} \cdot \lambda d^{\prime \prime} . d^{\prime \prime} x^{\prime \prime}\right) d\right) d d^{\prime \prime \prime}\right) d
\end{aligned}
$$

A program $p$ is translated as $\llbracket p \rrbracket^{\prime \prime}(\lambda x . \lambda d . d x) \lambda x . x$. 
Analysis: The simulation of literals, variables, and applications is standard. The control continuation of the body of each $\lambda$-abstraction is delimited, corresponding to it being evaluated with an empty control stack in the SECD machine. The J operator abstracts the control continuation and the dump continuation and immediately restores them, resuming the computation with a state appender which holds the abstracted dump continuation captive. Applying this state appender to a value $v$ yields a program closure (boxed in the three simulations above). Applying this program closure to a value $v^{\prime}$ has the effect of discarding both the current control continuation and the current dump continuation, applying $v$ to $v^{\prime}$, and resuming the captured dump continuation with the result.

The first rational deconstruction [9] already characterized the SECD machine in terms of the CPS hierarchy: the control stack is the first continuation, the dump is the second one (i.e., the meta-continuation), and abstraction bodies are evaluated within a control delimiter (i.e., an empty control stack). Our work further characterizes the $\mathrm{J}$ operator as capturing (a copy of) the metacontinuation.

\section{Four more simulations of the $\mathrm{J}$ operator}

\subsection{The $\mathcal{C}$ operator and the CPS hierarchy}

In the terminology of reflective towers [12], continuations captured with shift are "pushy" - at their point of invocation, they compose with the current continuation by "pushing" it on the meta-continuation. In the second encoding of $\mathrm{J}$ in Section 3, the term $\mathcal{S} \lambda d^{\prime} . d\left(x x^{\prime} \lambda x^{\prime \prime} . x^{\prime \prime}\right)$ serves to discard the current continuation $d^{\prime}$ before applying the captured continuation $d$. Because of this use of shift to discard $d^{\prime}$, the continuation $d$ is composed with the identity continuation.

On the other hand, still using the terminology of reflective towers, continuations captured with call/cc [8] or with Felleisen's $\mathcal{C}$ operator [15] are "jumpy"at their point of invocation, they discard the current continuation. If the continuation $d$ were captured with $\mathcal{C}$, then the term $d\left(x x^{\prime} \lambda x^{\prime \prime} . x^{\prime \prime}\right)$ would suffice to discard the current continuation.

The first encoding of $\mathrm{J}$ in Section 3 uses the pushy control operators $\mathcal{S}_{1}$ (i.e., $\mathcal{S}$ ) and $\mathcal{S}_{2}$. Murthy [33] and Kameyama [23] have investigated their jumpy counterparts in the CPS hierarchy, $\mathcal{C}_{1}$ (i.e., $\mathcal{C}$ ) and $\mathcal{C}_{2}$. Jumpy continuations therefore suggest two new simulations of the J operator. We show only the clauses for J, which are the only ones that change compared to Section 3. As before, we assume a call-by-value meta-language with right-to-left evaluation.

- In direct style, using $\mathcal{C}_{2}$, reset $2\left(\langle\cdot\rangle_{2}\right), \mathcal{C}_{1}$, and $\operatorname{reset}_{1}\left(\langle\cdot\rangle_{1}\right)$ :

$$
\llbracket \mathbf{J} \rrbracket=\mathcal{C}_{1} \lambda c . \mathcal{C}_{2} \lambda d . d\left(c \lambda x . \lambda x^{\prime} . d\left\langle x x^{\prime}\right\rangle_{1}\right)
$$

This simulation provides a new example of programming in the CPS hierarchy with jumpy delimited continuations. 
- In CPS with one layer of continuations, using $\mathcal{C}$ and reset $(\langle\cdot\rangle)$ :

$$
\llbracket \mathbf{J} \rrbracket^{\prime}=\lambda c . \mathcal{C} \lambda d . d\left(c \lambda x . \lambda c . c \lambda x^{\prime} \cdot \lambda c^{\prime} . d\left(x x^{\prime} \lambda x^{\prime \prime} . x^{\prime \prime}\right)\right)
$$

The corresponding CPS simulation of J with two layers of continuations coincides with the one in Section 3.

\subsection{The call/cc operator and the CPS hierarchy}

Like shift and $\mathcal{C}$, call/cc takes a snapshot of the current context. However, unlike shift and $\mathcal{C}$, in so doing call/cc leaves the current context in place. So for example, $1+($ call/cc $\lambda k .10)$ yields 11 because call/cc leaves the context $1+[$.$] in place, whereas both 1+(\mathcal{S} \lambda k .10)$ and $1+(\mathcal{C} \lambda k .10)$ yield 10 because the context $1+[$.$] is tossed away.$

Therefore $\mathrm{J}$ can be simulated in CPS with one layer of continuations, using call/cc and exploiting its non-abortive behavior:

$$
\llbracket \mathbf{J}^{\prime}=\lambda c . \text { call } / \mathbf{c c} \lambda d . c \lambda x . \lambda c . c \lambda x^{\prime} \cdot \lambda c^{\prime} . d\left(x x^{\prime} \lambda x^{\prime \prime} . x^{\prime \prime}\right)
$$

The obvious generalization of call/cc to the CPS hierarchy does not fit the bill as well, however. One needs an abort operator as well in order for call $/ \mathrm{cc}_{2}$ to capture the initial continuation and the current meta-continuation. We leave the rest of this train of thoughts to the imagination of the reader.

\section{On the design of control operators}

We note that replacing $\mathcal{C}$ with $\mathcal{S}$ above (resp. $\mathcal{C}_{1}$ with $\mathcal{S}_{1}$ and $\mathcal{C}_{2}$ with $\mathcal{S}_{2}$ ) yields a pushy counterpart for J, i.e., program closures returning to their point of activation. (Similarly, replacing $\mathcal{C}$ with $\mathcal{S}$ in the specification of call/cc in terms of $\mathcal{C}$ yields a pushy version of call/cc, assuming a global control delimiter.) One can also envision an abortive version of $\mathrm{J}$ that tosses away the context it abstracts. In that sense, control operators are easy to invent, though not always easy to implement efficiently. Nowadays, however, the acid test for a new control operator lies elsewhere, for example:

1. Which programming idiom does this control operator reflect?

2. What is the logical content of this control operator [22]?

Even though it was the first control operator ever, J passes this acid test. As pointed out by Thielecke,

1. $\mathrm{J}$ is a generalized return [39, Section 2.1], and

2. the type of $\mathbf{J} \lambda x . x$ is the law of the excluded middle [40, Section 5.2]. 
On the other hand, despite its remarkable fit to Algol labels and jumps [16,28], $\mathrm{J}$ is unintuitive to use. For example, if a let expression is the syntactic sugar of a beta-redex (and $x_{1}$ is fresh), the equivalence

$$
t_{0} t_{1} \cong \text { let } x_{1}=t_{1} \text { in } t_{0} x_{1}
$$

does not hold in the presence of J, even though it does in the presence of call/cc, $\mathcal{C}$, and shift for right-to-left evaluation.

\section{Related work}

\subsection{Landin and Burge}

Landin [27] introduced the $\mathrm{J}$ operator as a new language feature motivated by three questions about labels and jumps:

- Can a language have jumps without having assignment?

- Is there some component of jumping that is independent of labels?

- Is there some feature that corresponds to functions with arguments in the same sense that labels correspond to procedures without arguments?

He gave the semantics of the $\mathrm{J}$ operator by extending the SECD machine. In addition to using $\mathrm{J}$ to model jumps in Algol 60 [26], he gave examples of programming with the $\mathrm{J}$ operator, using it to represent failure actions as program closures where it is essential that they abandon the context of their application.

In his textbook [6, Section 2.10], Burge adjusted Landin's original specification of the J operator. Indeed, in Landin's extension of the SECD machine, $\mathrm{J}$ could only occur in the context of an application. Burge adjusted the original specification so that $\mathrm{J}$ could occur in arbitrary contexts. To this end, he introduced the notion of a "state appender" as the denotation of J.

Thielecke [39] gave a detailed introduction to the $\mathrm{J}$ operator as presented by Landin and Burge. Burstall [7] illustrated the use of the $\mathrm{J}$ operator by simulating threads for parallel search algorithms, which in retrospect is the first simulation of threads in terms of first-class continuations.

\subsection{Reynolds}

Reynolds [34] gave a comparison of $J$ to escape, the binder form of Scheme's call/cc [8]. ${ }^{1}$ He gave encodings of Landin's J (i.e., restricted to the context of an application) and escape in terms of each other.

His encoding of escape in terms of $\mathrm{J}$ reads as follows:

$$
(\text { escape } k \text { in } t)^{*}=\operatorname{let} k=\mathbf{J} \lambda x . x \text { in } t^{*}
$$

As Thielecke notes [39], this encoding is only valid immediately inside an abstraction. Indeed, the dump continuation captured by $\mathrm{J}$ only coincides with

\footnotetext{
${ }^{1}$ escape $k$ in $t \equiv$ call $/$ cc $\lambda k . t$
} 
the continuation captured by escape if the control continuation is the initial one (i.e., immediately inside a control delimiter). Thielecke generalized the encoding by adding a dummy abstraction:

$$
(\text { escape } k \text { in } t)^{*}=\left(\lambda() . \operatorname{let} k=\mathbf{J} \lambda x . x \text { in } t^{*}\right)()
$$

From the point of view of the rational deconstruction, this dummy abstraction implicitly inserts a control delimiter.

Reynolds's converse encoding of $\mathrm{J}$ in terms of escape reads as follows:

$$
\left(\text { let } d=\mathbf{J} \lambda x . t_{1} \text { in } t_{0}\right)^{\circ}=\text { escape } k \text { in }\left(\operatorname{let} d=\lambda x . k t_{1}^{\circ} \text { in } t_{0}{ }^{\circ}\right)
$$

where $k$ does not occur free in $t_{0}$ and $t_{1}$. For the same reason as above, this encoding is only valid immediately inside an abstraction.

\subsection{Felleisen}

Felleisen showed how to embed Landin's extension of applicative expressions with $\mathrm{J}$ into the Scheme programming language [16]. The embedding is defined as Scheme syntactic extensions (i.e., macros). J is treated as a dynamic identifier that is bound in the body of every abstraction. Its control aspect is handled through Scheme's control operator call/cc.

As pointed out by Thielecke [39], Felleisen's simulation can be stated in direct style, assuming a call-by-value meta-language with right-to-left evaluation and call/cc. In addition, we present the corresponding simulations using $\mathcal{C}$ and reset, using shift and reset, and in CPS:

- In direct style, using either of call/cc, $\mathcal{C}$, or shift $(\mathcal{S})$, and one global control delimiter $(\langle\cdot\rangle)$ :

$$
\begin{aligned}
\llbracket x \rrbracket & =x \\
\llbracket t_{0} t_{1} \rrbracket & =\llbracket t_{0} \rrbracket \llbracket t_{1} \rrbracket \\
\llbracket \lambda x . t \rrbracket & =\lambda x . \text { call } / \text { cc } \lambda d \cdot \text { let } \mathbf{J}=\lambda x \cdot \lambda x^{\prime} . d\left(x x^{\prime}\right) \llbracket \text { in } \llbracket t \rrbracket \\
& =\lambda x \cdot \mathcal{C} \lambda d . \text { let } \mathbf{J}=\lambda x \cdot \lambda x^{\prime} \cdot d\left(x x^{\prime}\right) \text { in } d \llbracket t \rrbracket \\
& =\lambda x \cdot \mathcal{S} \lambda d . \text { let } \mathbf{J}=\lambda x \cdot \lambda x^{\prime} \cdot \mathcal{S} \lambda c^{\prime} \cdot d\left(x x^{\prime}\right) \text { in } d \llbracket t \rrbracket
\end{aligned}
$$

A program $p$ is translated as $\langle\llbracket p \rrbracket\rangle$.

- In CPS:

$$
\begin{aligned}
\llbracket x \rrbracket^{\prime} & =\lambda c . c x \\
\llbracket t_{0} t_{1} \rrbracket^{\prime} & =\lambda c . \llbracket t_{1} \rrbracket^{\prime} \lambda x_{1} \cdot \llbracket t_{0} \rrbracket^{\prime} \lambda x_{0} \cdot x_{0} x_{1} c \\
\llbracket \lambda x . t \rrbracket^{\prime} & =\lambda c . c\left(\lambda x . \lambda d . \text { let } \mathbf{J}=\lambda x . \lambda c . c \lambda \lambda x^{\prime} \cdot \lambda c^{\prime} \cdot x x^{\prime} d\right.
\end{aligned}
$$

A program $p$ is translated as $\llbracket p \rrbracket^{\prime} \lambda x . x$. 
Analysis: The simulation of variables and applications is standard. The continuation of the body of each $\lambda$-abstraction is captured, and the identifier $\mathrm{J}$ is dynamically bound to a function closure (the state appender) which holds the continuation captive. Applying this function closure to a value $v$ yields a program closure (boxed in the simulations above). Applying this program closure to a value $v^{\prime}$ has the effect of applying $v$ to $v^{\prime}$ and resuming the captured continuation with the result, abandoning the current continuation.

\subsection{Felleisen and Burge}

Felleisen's version of the SECD machine with the J operator differs from Burge's. In the notation of Section 2.1, Burge's clause for applying program closures reads

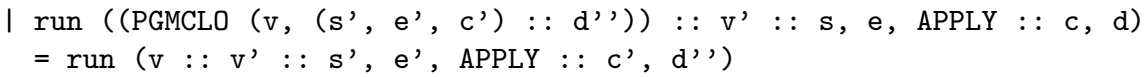

instead of

I run ((PGMCLO (v, d')) : : v' : : s, e, APPLY :: c, d)

$=\operatorname{run}\left(\mathrm{v}:: \mathrm{v}^{\prime}::\right.$ nil, e_init, APPLY $::$ nil, d')

Felleisen's version delays the consumption of the dump until the function, in the program closure, completes, whereas Burge's version does not. The modification is unobservable because a program cannot capture the control continuation and because applying the argument of a state appender pushes the data stack, the environment, and the control stack on the dump. Felleisen's modification can be characterized as wrapping a control delimiter around the argument of a dump continuation, similarly to the simulation of static delimited continuations in terms of dynamic ones [5].

Burge's version, however, is not in defunctionalized form. In Section 7, we put it in defunctionalized form without inserting a control delimiter and we outline the corresponding compositional evaluation functions and simulations.

\section{An alternative deconstruction}

\subsection{Our starting point: Burge's specification}

As pointed out in Section 6.4, Felleisen's version of the SECD machine applies the value contained in a program closure before restoring the components of the captured dump. Burge's version differs by restoring the components of the captured dump before applying the value contained in the program closure. In other words,

- Felleisen's version applies the value contained in a program closure with an empty data stack, a dummy environment, an empty control stack, and the captured dump, whereas

- Burge's version applies the value contained in a program closure with the captured data stack, environment, control stack, and previous dump. 
The versions induce a minor programming difference because the first makes it possible to use $\mathrm{J}$ in any context whereas the second restricts $\mathrm{J}$ to occur only inside a $\lambda$-abstraction.

Burge's specification of the SECD machine with $\mathrm{J}$ follows. Ellipses mark what does not change from the specification of Section 2.1:

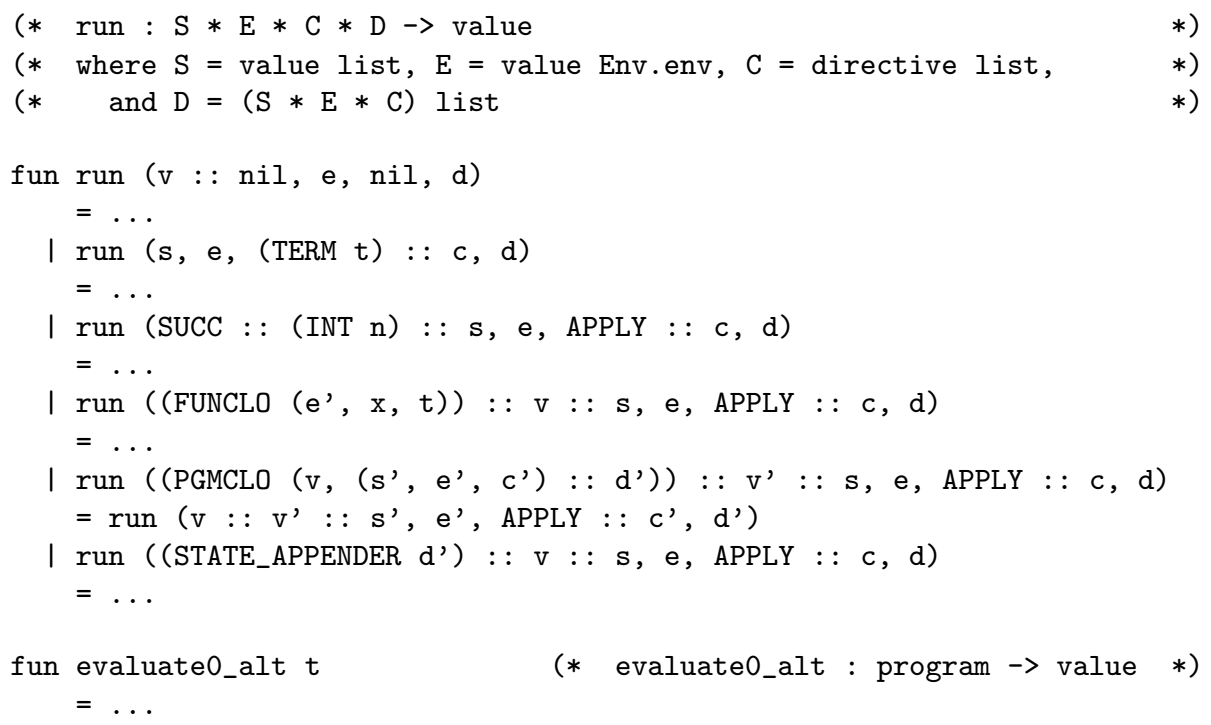

Just as in Section 2.2, Burge's specification can be disentangled into four mutuallyrecursive transition functions. The disentangled specification, however, is not in defunctionalized form. We put it next in defunctionalized form without inserting a control delimiter, and then repeat the rest of the rational deconstruction.

\subsection{Burge's specification in defunctionalized form}

The disentangled specification of Burge is not in defunctionalized form because the dump does not have a single point of consumption. It is consumed by run_d for values yielded by the body of $\lambda$-abstractions and in run_a for values thrown to program closures. In order to be in the image of Reynolds's defunctionalization and have run_d as the apply function, the dump should be solely consumed by run_d. We therefore distinguish values yielded by normal evaluation and values thrown to program closures, and we make run_d dispatch on these two kinds of returned values. For values yielded by normal evaluation (i.e., in the call from run_c to run_d), run_d proceeds as before. For values thrown to program closures, run_d calls run_a. Our modification therefore adds one transition (from run_a to run_d) for values thrown to program closures.

The change only concerns three clauses and ellipses mark what does not change from the evaluator of Section 2.2:

datatype returned_value $=$ YIELD of value

I THROW of value * value 


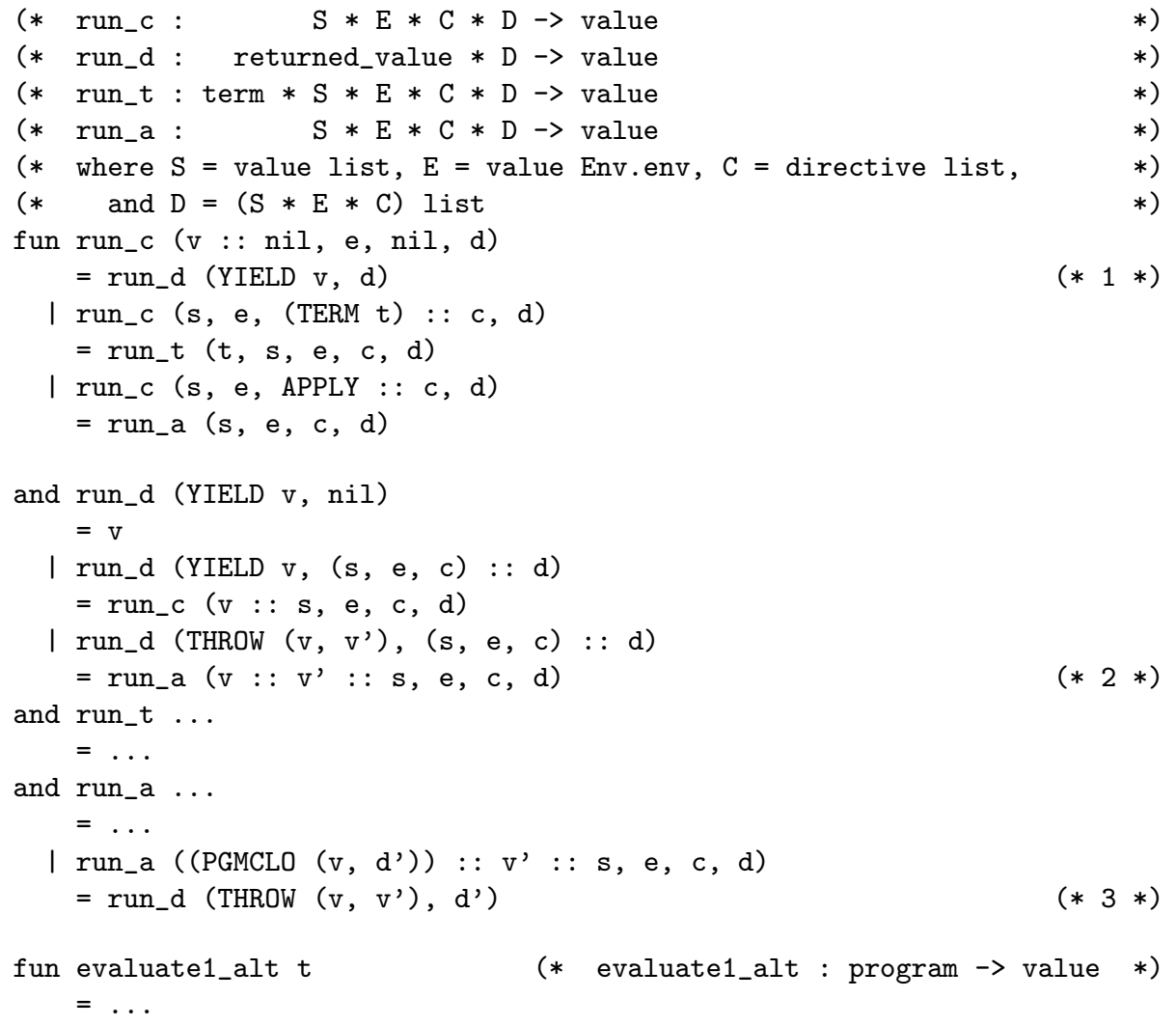

YIELD is used to tag values returned by function closures (in the clause marked "1" above), and THROW is used to tag values sent to program closures (in the clause marked " 3 "). THROW tags a pair of values, which will be applied in run_d (by calling run_a in the clause marked "2").

Proposition 7 (full correctness) Given a program, evaluate0_alt and evaluate1_alt either both diverge or both yield values that are structurally equal.

\subsection{A higher-order counterpart}

In the modified specification of Section 7.2, the data types of control stacks and dumps are identical to those of the disentangled machine of Section 2.2. These data types, together with run_d and run_c, are in the image of defunctionalization (run_d and run_c are their apply functions). The corresponding higher-order evaluator reads as follows:

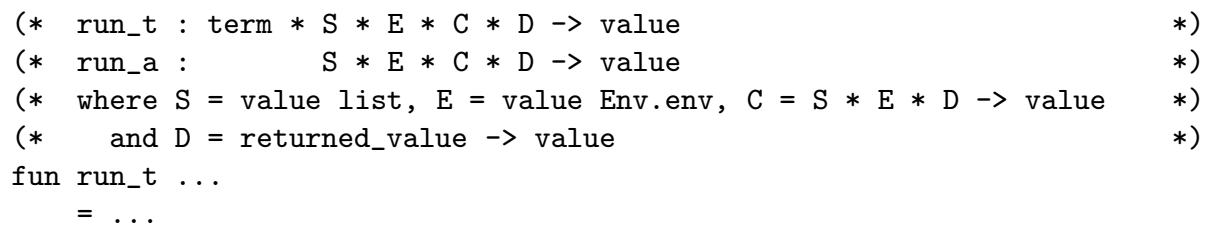




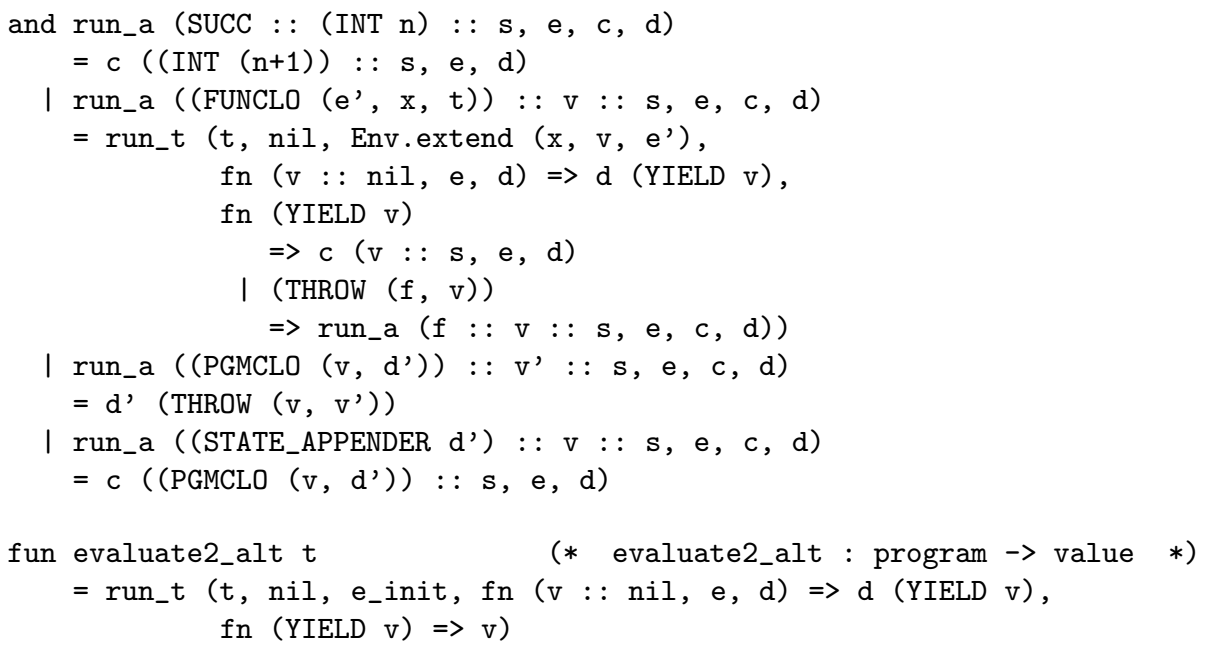

As before, the resulting evaluator is in continuation-passing style (CPS), with two layered continuations. It threads a stack of intermediate results, a (calleesave) environment, a control continuation, and a dump continuation. The values sent to dump continuations are tagged to indicate whether they represent the result of a function closure or an application of a program closure. Defunctionalizing this evaluator yields the definition of Section 7.2.

Proposition 8 (full correctness) Given a program, evaluate1_alt and evaluate2_alt either both diverge or yield expressible values that are structurally equal.

\subsection{The rest of the rational deconstruction}

The evaluator of Section 7.3 can be transformed exactly as the higher-order evaluator of Section 2.3:

1. Eliminating the data stack and the callee-save environment yields a traditional eval-apply evaluator, with run_t as eval and run_a as apply. The evaluator is in CPS with two layers of continuations.

2. Direct-style transformation with respect to the dump yields an evaluator that uses shift and reset (or $\mathcal{C}$ and a global reset, or again call/cc and a global reset) to manipulate the implicit dump continuation.

3. A second direct-style transformation with respect to the control stack yields an evaluator in direct style that uses the delimited-control operators

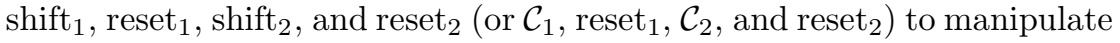
the implicit control and dump continuations.

4. Refunctionalizing the applicable values yields a compositional, higherorder, direct-style evaluator corresponding to Burge's specification of the J operator. 


\subsection{Three alternative simulations of the $\mathrm{J}$ operator}

As in Section 3, the compositional counterpart of the evaluators of Section 7.4 can be viewed as syntax-directed encodings into their meta-language. Below, we state these encodings as three simulations of J: one in direct style, one in CPS with one layer of continuations, and one in CPS with two layers of continuations.

Again, we assume a call-by-value meta-language with right-to-left evaluation and with a sum (to distinguish values returned by functions and values sent to program closures), a case expression (for the body of $\lambda$-abstractions) and a destructuring let expression (at the top level).

- In direct style, using either of shift $_{2}$, reset $_{2}$, shift $_{1}$, and reset $_{1}$ or of $\mathcal{C}_{2}$, reset $_{2}, \mathcal{C}_{1}$, and reset $_{1}$ :

$$
\begin{aligned}
& \llbracket n \rrbracket=n \\
& \llbracket x \rrbracket=x \\
& \llbracket t_{0} t_{1} \rrbracket=\llbracket t_{0} \rrbracket \llbracket t_{1} \rrbracket \\
& \llbracket \lambda x . t \rrbracket=\lambda x \text {.case } \quad\langle\operatorname{inL} \llbracket t \rrbracket\rangle_{1} \\
& \text { of } \operatorname{inL}(x) \Rightarrow x \\
& \mid \operatorname{inR}\left(x, x^{\prime}\right) \Rightarrow x x^{\prime} \\
& \llbracket \mathbf{J} \rrbracket=\mathcal{S}_{1} \lambda c . \mathcal{S}_{2} \lambda d . d\left(c \lambda x . \lambda x^{\prime} \cdot \mathcal{S}_{1} \lambda c^{\prime} \cdot \mathcal{S}_{2} \lambda d^{\prime} . d\left(\mathbf{i n R}\left(x, x^{\prime}\right)\right)\right) \\
& =\mathcal{C}_{1} \lambda c \cdot \mathcal{C}_{2} \lambda d . d\left(c \lambda x, \lambda x^{\prime} \cdot d\left(\mathbf{i n R}\left(x, x^{\prime}\right)\right)\right)
\end{aligned}
$$

A program $p$ is translated as $\left\langle\text { let } \operatorname{inL}(x)=\langle\operatorname{inL}(\llbracket p \rrbracket)\rangle_{1} \text { in } x\right\rangle_{2}$.

- In CPS with one layer of continuations, using either of shift and reset, of $\mathcal{C}$ and reset, or of call/cc and reset:

$$
\begin{aligned}
& \llbracket n \rrbracket^{\prime}=\lambda c . c n \\
& \llbracket x \rrbracket^{\prime}=\lambda \text { c.c } x \\
& \llbracket t_{0} t_{1} \rrbracket^{\prime}=\lambda c \cdot \llbracket t_{1} \rrbracket^{\prime}\left(\lambda x_{1} \cdot \llbracket t_{0} \rrbracket^{\prime} \lambda x_{0} \cdot x_{0} x_{1} c\right) \\
& \llbracket \lambda x . t \rrbracket^{\prime}=\lambda c . c\left(\lambda x . \lambda c . \text { case } \llbracket t \rrbracket^{\prime} \lambda x . \mathbf{i n L}(x)\right. \\
& \text { of } \operatorname{inL}(x) \Rightarrow c x \\
& \left.\mid \operatorname{inR}\left(x, x^{\prime}\right) \Rightarrow x x^{\prime} c\right) \\
& \llbracket \mathbf{J} \rrbracket^{\prime}=\lambda c . \mathcal{S} \lambda d . d\left(c \lambda x . \lambda c . c \lambda \lambda x^{\prime} \cdot \lambda c^{\prime} \cdot \mathcal{S} \lambda d^{\prime} . d\left(\mathbf{i n R}\left(x, x^{\prime}\right)\right)\right. \\
& =\lambda c \cdot \mathcal{C} \lambda d \cdot d\left(c \lambda x . \lambda c \cdot c \overline{\left.\lambda x^{\prime} \cdot \lambda c^{\prime} \cdot d\left(\mathbf{i n R}\left(x, x^{\prime}\right)\right)\right)}\right. \\
& =\lambda c . \text { call } / \mathbf{c c} \lambda d . c \lambda x . \lambda c . c \lambda x^{\prime} . \lambda c^{\prime} . d\left(\operatorname{inR}\left(x, x^{\prime}\right)\right)
\end{aligned}
$$

A program $p$ is translated as $\left\langle\operatorname{let} \operatorname{inL}(x)=\llbracket p \rrbracket^{\prime} \lambda x \cdot \operatorname{inL}(x)\right.$ in $\left.x\right\rangle$.

- In CPS with two layers of continuations:

$$
\begin{aligned}
& \llbracket n \rrbracket^{\prime \prime}=\lambda c . \lambda d . c n d \\
& \llbracket x \rrbracket^{\prime \prime}=\lambda c . \lambda \text { d.c } x d \\
& \llbracket t_{0} t_{1} \rrbracket^{\prime \prime}=\lambda c \cdot \lambda d \cdot \llbracket t_{1} \rrbracket^{\prime \prime}\left(\lambda x_{1} \cdot \lambda d \cdot \llbracket t_{0} \rrbracket^{\prime \prime}\left(\lambda x_{0} \cdot \lambda d \cdot x_{0} x_{1} c d\right) d\right) d \\
& \llbracket \lambda x . t \rrbracket^{\prime \prime}=\lambda c . \lambda d . c\left(\lambda x . \lambda c . \lambda d . \llbracket t \rrbracket^{\prime \prime}(\lambda x . \lambda d . d(\operatorname{inL}(x)))\right. \\
& \lambda x^{\prime \prime} \text {.case } x^{\prime \prime} \\
& \text { of } \operatorname{inL}(x) \Rightarrow c x d \\
& \text { | } \left.\operatorname{inR}\left(x, x^{\prime}\right) \Rightarrow x x^{\prime} c d\right) d \\
& \llbracket \mathbf{J} \rrbracket^{\prime \prime}=\lambda c . \lambda d . c\left(\lambda x \cdot \lambda c \cdot \lambda d^{\prime \prime \prime} . c\left(\lambda x^{\prime} \cdot \lambda c^{\prime} \cdot \lambda d^{\prime} . d\left(\mathbf{i n R}\left(x, x^{\prime}\right)\right)\right) d d^{\prime \prime \prime}\right) d
\end{aligned}
$$




$$
\begin{aligned}
\text { A program } p \text { is translated as } \llbracket p \rrbracket^{\prime \prime} & (\lambda x . \lambda d . d(\operatorname{inL}(x))) \\
& \lambda x \cdot \operatorname{let} \operatorname{inL}\left(x^{\prime}\right)=x \operatorname{in} x^{\prime} .
\end{aligned}
$$

Analysis: The simulation of literals, variables, and applications is standard. The body of each $\lambda$-abstraction is evaluated with a control continuation injecting the resulting value into the sum type to indicate normal completion and resuming the current dump continuation, and with a dump continuation inspecting the resulting sum to determine whether to continue normally or to apply a program closure. Continuing normally consists of invoking the control continuation with the resulting value and the dump continuation. Applying a program closure consists of restoring the components of the dump and then performing the application. The $\mathrm{J}$ operator abstracts both the control continuation and the dump continuation and immediately restores them, resuming the computation with a state appender holding the abstracted dump continuation captive. Applying this state appender to a value $v$ yields a program closure (boxed in the three simulations above). Applying this program closure to a value $v^{\prime}$ has the effect of discarding both the current control continuation and the current dump continuation, injecting $v$ and $v^{\prime}$ into the sum type to indicate exceptional completion, and resuming the captured dump continuation. It is an error to evaluate $\mathrm{J}$ outside of a $\lambda$-abstraction.

\subsection{Related work}

Kiselyov's encoding of dynamic delimited continuations in terms of the static delimited-continuation operators shift and reset [24] is similar to this alternative encoding of the $\mathrm{J}$ operator. Both encodings tag the argument to the metacontinuation to indicate whether it represents a normal return or a value thrown to a first-class continuation. However, in order to encode dynamic delimited continuations, Kiselyov uses a recursive meta-continuation.

\section{Summary and conclusion}

We have extended the rational deconstruction of the SECD machine to the J operator, and we have presented a series of alternative implementations, including a compositional evaluation function in CPS. In passing, we have also presented new applications of defunctionalization and new examples of control delimiters and of both pushy and jumpy delimited continuations in programming practice. 


\section{On the origin of first-class continuations}

We have shown that jumping and labels are not essentially connected with strings of imperatives and in particular, with assignment. Second, that jumping is not essentially connected with labels. In performing this piece of logical analysis we have provided a precisely limited sense in which the "value of a label" has meaning. Also, we have discovered a new language feature, not present in current programming languages, that promises to clarify and simplify a notoriously untidy area of programming - that concerned with success/failure situations, and the actions needed on failure. - Peter J. Landin, 1965 [27, page 133]

It was Strachey who coined the term "first-class functions" [38, Section 3.5.1]. ${ }^{2}$ In turn it was Landin who, through the J operator, invented what we know today as first-class continuations [18]. Indeed, like Reynolds for escape, Landin defined $\mathrm{J}$ in an unconstrained way, i.e., with no regard for it to be compatible with the last-in, first-out allocation discipline prevalent for control stacks since Algol 60. ${ }^{3}$

Today, 'continuations' is an overloaded term, that may refer

- to the original semantic description technique for representing 'the meaning of the rest of the program' as a function, the continuation, as multiply co-discovered at the turn of the 1970's [35]; or

- to the programming-language feature of first-class continuations as typically provided by a control operator such as J, escape, or call/cc, as invented by Landin.

Whether a semantic description technique or a programming-language feature, the goal of continuations was the same: to formalize Algol's labels and jumps. But where Wadsworth and Abdali gave a continuation semantics to Algol, Landin translated Algol programs into applicative expressions in direct style. In turn, he specified the semantics of applicative expressions with the SECD machine, i.e., using first-order means. The meaning of an Algol label was an ISWIM 'program closure' as obtained by the J operator. Program closures were defined by extending the SECD machine, i.e., still using first-order means.

Landin did not use an explicit representation of the rest of the computation in his direct semantics of Algol 60, and so he is not listed among the co-discoverers of continuations [35]. Such an explicit representation, however, exists in the SECD machine, in first-order form: the dump, which represents the rest of the computation after returning from the current function call.

In this article, we have shown that, though it is first-order, the SECD machine directly corresponds to a compositional evaluation function in CPS - the tool of choice for specifying control operators since Reynolds's work [34]. As a corollary, the dump directly corresponds to a functional representation of control, since it is a defunctionalized continuation. Therefore, in the light of defunctionalization, we wish to see Landin's name added to the list of co-discoverers of continuations.

\footnotetext{
2 "Out of Quine's dictum: To be is to be the value of a variable, grew Strachey's 'first-class citizens'." Peter J. Landin, 2000 [30, page 75]

3 "Dumps and program-closures are data-items, with all the implied latency for unruly multiple use and other privileges of first-class-citizenship." Peter J. Landin, 1997 [29, Section 1]
} 
Acknowledgments: Thanks are due to Dariusz Biernacki, Julia L. Lawall, Kristian Støvring, and the anonymous reviewers of IFL'05 for comments. We are also grateful to Andrzej Filinski, Dan Friedman, Lockwood Morris, John Reynolds, Guy Steele, Carolyn Talcott, Bob Tennent, Hayo Thielecke, and Chris Wadsworth for their feedback on Section 9.

This work was partly carried out while the two authors visited the TOPPS group at DIKU (http://www.diku.dk/topps). It is partly supported by the Danish Natural Science Research Council, Grant no. 21-03-0545 and by the ESPRIT Working Group APPSEM II (http://www.appsem.org).

\section{References}

[1] Mads Sig Ager, Dariusz Biernacki, Olivier Danvy, and Jan Midtgaard. A functional correspondence between evaluators and abstract machines. In Dale Miller, editor, Proceedings of the Fifth ACM-SIGPLAN International Conference on Principles and Practice of Declarative Programming (PPDP'03), pages 8-19. ACM Press, August 2003.

[2] Mads Sig Ager, Olivier Danvy, and Jan Midtgaard. A functional correspondence between call-by-need evaluators and lazy abstract machines. Information Processing Letters, 90(5):223-232, 2004. Extended version available as the technical report BRICS RS-04-3.

[3] Mads Sig Ager, Olivier Danvy, and Jan Midtgaard. A functional correspondence between monadic evaluators and abstract machines for languages with computational effects. Theoretical Computer Science, 342(1):149-172, 2005. Extended version available as the technical report BRICS RS-04-28.

[4] Małgorzata Biernacka, Dariusz Biernacki, and Olivier Danvy. An operational foundation for delimited continuations in the CPS hierarchy. Logical Methods in Computer Science, 1(2:5):1-39, November 2005. A preliminary version was presented at the Fourth ACM SIGPLAN Workshop on Continuations (CW'04).

[5] Dariusz Biernacki and Olivier Danvy. A simple proof of a folklore theorem about delimited control. Research Report BRICS RS-05-25, DAIMI, Department of Computer Science, University of Aarhus, Aarhus, Denmark, August 2005. Theoretical Pearl to appear in the Journal of Functional Programming.

[6] William H. Burge. Recursive Programming Techniques. Addison-Wesley, 1975.

[7] Rod M. Burstall. Writing search algorithms in functional form. In Donald Michie, editor, Machine Intelligence, volume 5, pages 373-385. Edinburgh University Press, 1969.

[8] William Clinger, Daniel P. Friedman, and Mitchell Wand. A scheme for a higher-level semantic algebra. In John Reynolds and Maurice Nivat, edi- 
tors, Algebraic Methods in Semantics, pages 237-250. Cambridge University Press, 1985.

[9] Olivier Danvy. A rational deconstruction of Landin's SECD machine. In Clemens Grelck, Frank Huch, Greg J. Michaelson, and Phil Trinder, editors, Implementation and Application of Functional Languages, 16th International Workshop, IFL'04, number 3474 in Lecture Notes in Computer Science, pages 52-71, Lübeck, Germany, September 2004. Springer-Verlag. Recipient of the 2004 Peter Landin prize. Extended version available as the technical report BRICS RS-03-33.

[10] Olivier Danvy and Andrzej Filinski. Abstracting control. In Mitchell Wand, editor, Proceedings of the 1990 ACM Conference on Lisp and Functional Programming, pages 151-160, Nice, France, June 1990. ACM Press.

[11] Olivier Danvy and Julia L. Lawall. Back to direct style II: First-class continuations. In William Clinger, editor, Proceedings of the 1992 ACM Conference on Lisp and Functional Programming, LISP Pointers, Vol. V, No. 1, pages 299-310, San Francisco, California, June 1992. ACM Press.

[12] Olivier Danvy and Karoline Malmkjær. Intensions and extensions in a reflective tower. In Robert (Corky) Cartwright, editor, Proceedings of the 1988 ACM Conference on Lisp and Functional Programming, pages 327341, Snowbird, Utah, July 1988. ACM Press.

[13] Olivier Danvy and Lasse R. Nielsen. Defunctionalization at work. In Harald Søndergaard, editor, Proceedings of the Third International ACM SIGPLAN Conference on Principles and Practice of Declarative Programming (PPDP'01), pages 162-174, Firenze, Italy, September 2001. ACM Press. Extended version available as the technical report BRICS RS-01-23.

[14] Olivier Danvy and Zhe Yang. An operational investigation of the CPS hierarchy. In S. Doaitse Swierstra, editor, Proceedings of the Eighth European Symposium on Programming, number 1576 in Lecture Notes in Computer Science, pages 224-242, Amsterdam, The Netherlands, March 1999. Springer-Verlag.

[15] Matthias Felleisen. The Calculi of $\lambda$-v-CS Conversion: A Syntactic Theory of Control and State in Imperative Higher-Order Programming Languages. $\mathrm{PhD}$ thesis, Computer Science Department, Indiana University, Bloomington, Indiana, August 1987.

[16] Matthias Felleisen. Reflections on Landin's J operator: a partly historical note. Computer Languages, 12(3/4):197-207, 1987.

[17] Andrzej Filinski. Representing monads. In Hans-J. Boehm, editor, Proceedings of the Twenty-First Annual ACM Symposium on Principles of Programming Languages, pages 446-457, Portland, Oregon, January 1994. ACM Press. 
[18] Daniel P. Friedman and Christopher T. Haynes. Constraining control. In Mary S. Van Deusen and Zvi Galil, editors, Proceedings of the Twelfth Annual ACM Symposium on Principles of Programming Languages, pages 245-254, New Orleans, Louisiana, January 1985. ACM Press.

[19] Daniel P. Friedman, Mitchell Wand, and Christopher T. Haynes. Essentials of Programming Languages, second edition. The MIT Press, 2001.

[20] Yoshihiko Futamura. Partial evaluation of computation process - an approach to a compiler-compiler. Systems - Computers - Controls, 2(5):45-50, 1971. Reprinted in Higher-Order and Symbolic Computation 12(4):381391, 1999, with an interview [21].

[21] Yoshihiko Futamura. Partial evaluation of computation process, revisited. Higher-Order and Symbolic Computation, 12(4):377-380, 1999.

[22] Timothy G. Griffin. A formulae-as-types notion of control. In Paul Hudak, editor, Proceedings of the Seventeenth Annual ACM Symposium on Principles of Programming Languages, pages 47-58, San Francisco, California, January 1990. ACM Press.

[23] Yukiyoshi Kameyama. Axioms for delimited continuations in the CPS hierarchy. In Jerzy Marcinkowski and Andrzej Tarlecki, editors, Computer Science Logic, 18th International Workshop, CSL 2004, 13th Annual Conference of the EACSL, Proceedings, volume 3210 of Lecture Notes in Computer Science, pages 442-457, Karpacz, Poland, September 2004. Springer.

[24] Oleg Kiselyov. How to remove a dynamic prompt: Static and dynamic delimited continuation operators are equally expressible. Technical Report 611, Computer Science Department, Indiana University, Bloomington, Indiana, March 2005.

[25] Peter J. Landin. The mechanical evaluation of expressions. The Computer Journal, 6(4):308-320, 1964.

[26] Peter J. Landin. A correspondence between Algol 60 and Church's lambda notation. Communications of the ACM, 8:89-101 and 158-165, 1965.

[27] Peter J. Landin. A generalization of jumps and labels. Research report, UNIVAC Systems Programming Research, 1965. Reprinted in Higher-Order and Symbolic Computation 11(2):125-143, 1998, with a foreword [39].

[28] Peter J. Landin. The next 700 programming languages. Communications of the ACM, 9(3):157-166, 1966.

[29] Peter J. Landin. Histories of discoveries of continuations: Belles-lettres with equivocal tenses. In Olivier Danvy, editor, Proceedings of the Second ACM SIGPLAN Workshop on Continuations (CW'97), Technical report BRICS NS-96-13, University of Aarhus, pages 1:1-9, Paris, France, January 1997. 
[30] Peter J. Landin. My years with Strachey. Higher-Order and Symbolic Computation, 13(1/2):75-76, 2000.

[31] F. Lockwood Morris. The next 700 formal language descriptions. Lisp and Symbolic Computation, 6(3/4):249-258, 1993. Reprinted from a manuscript dated 1970 .

[32] Peter D. Mosses. A foreword to 'Fundamental concepts in programming languages'. Higher-Order and Symbolic Computation, 13(1/2):7-9, 2000.

[33] Chethan R. Murthy. Control operators, hierarchies, and pseudo-classical type systems: A-translation at work. In Olivier Danvy and Carolyn L. Talcott, editors, Proceedings of the First ACM SIGPLAN Workshop on Continuations (CW'92), Technical report STAN-CS-92-1426, Stanford University, pages 49-72, San Francisco, California, June 1992.

[34] John C. Reynolds. Definitional interpreters for higher-order programming languages. In Proceedings of 25th ACM National Conference, pages 717740, Boston, Massachusetts, 1972. Reprinted in Higher-Order and Symbolic Computation 11(4):363-397, 1998, with a foreword [36].

[35] John C. Reynolds. The discoveries of continuations. Lisp and Symbolic Computation, 6(3/4):233-247, 1993.

[36] John C. Reynolds. Definitional interpreters revisited. Higher-Order and Symbolic Computation, 11(4):355-361, 1998.

[37] Guy L. Steele Jr. Rabbit: A compiler for Scheme. Master's thesis, Artificial Intelligence Laboratory, Massachusetts Institute of Technology, Cambridge, Massachusetts, May 1978. Technical report AI-TR-474.

[38] Christopher Strachey. Fundamental concepts in programming languages. International Summer School in Computer Programming, Copenhagen, Denmark, August 1967. Reprinted in Higher-Order and Symbolic Computation 13(1/2):11-49, 2000, with a foreword [32].

[39] Hayo Thielecke. An introduction to Landin's "A generalization of jumps and labels". Higher-Order and Symbolic Computation, 11(2):117-124, 1998.

[40] Hayo Thielecke. Comparing control constructs by double-barrelled CPS. Higher-Order and Symbolic Computation, 15(2/3):141-160, 2002. 


\section{Recent BRICS Report Series Publications}

RS-06-4 Olivier Danvy and Kevin Millikin. A Rational Deconstruction of Landin's $J$ Operator. February 2006. ii +26 pp. To appear in the post-reviewed proceedings of the 17th International Workshop on the Implementation and Application of Functional Languages (IFL'05), Dublin, Ireland, September 2005.

RS-06-3 Małgorzata Biernacka and Olivier Danvy. A Concrete Framework for Environment Machines. February 2006 . ii+29 pp. To appear in the ACM Transactions on Computational Logic. Supersedes BRICS RS-05-15.

RS-06-2 Mikkel Baun Kjærgaard and Jonathan Bunde-Pedersen. A Formal Model for Context-Awareness. February 2006. 26 pp.

RS-06-1 Luca Aceto, Taolue Chen, Willem Jan Fokkink, and Anna Ingólfsdóttir. On the Axiomatizability of Priority. January 2006. 25 pp.

RS-05-38 Małgorzata Biernacka and Olivier Danvy. A Syntactic Correspondence between Context-Sensitive Calculi and Abstract Machines. December 2005. iii+39 pp. Revised version of BRICS RS-05-22.

RS-05-37 Gerth Stølting Brodal, Kanela Kaligosi, Irit Katriel, and Martin Kutz. Faster Algorithms for Computing Longest Common Increasing Subsequences. December 2005. 16 pp.

RS-05-36 Dariusz Biernacki, Olivier Danvy, and Chung-chieh Shan. On the Static and Dynamic Extents of Delimited Continuations. December 2005. ii+33 pp. To appear in the journal Science of Computer Programming. Supersedes BRICS RS-05-13.

RS-05-35 Kristian Støvring. Extending the Extensional Lambda Calculus with Surjective Pairing is Conservative. November 2005. 19 pp.

RS-05-34 Henning Korsholm Rohde. Formal Aspects of Polyvariant Specialization. November 2005. 27 pp.

RS-05-33 Luca Aceto, Willem Jan Fokkink, Anna Ingólfsdóttir, and Sumit Nain. Bisimilarity is not Finitely Based over BPA with Interrupt. October 2005. $33 \mathrm{pp}$. This paper supersedes BRICS Report RS-04-24. An extended abstract of this paper appeared in Algebra and Coalgebra in Computer Science, 1st Conference, CALCO 2005, Swansea, Wales, 3-6 September 2005, Lecture Notes in Computer Science 3629, pp. 54-68, Springer-Verlag, 2005. 